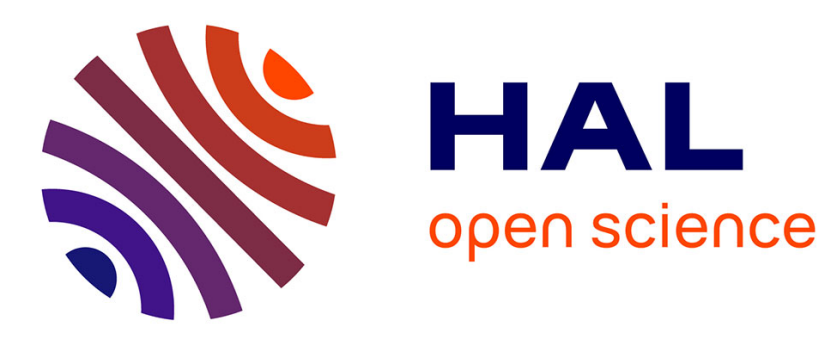

\title{
The SOS model partition function and the elliptic weight functions
}

\author{
Stanislav Pakuliak, Vladimir Roubtsov, Alexey Silantyev
}

\section{To cite this version:}

Stanislav Pakuliak, Vladimir Roubtsov, Alexey Silantyev. The SOS model partition function and the elliptic weight functions. Journal of Physics A: Mathematical and Theoretical, 2008, 41 (29), Non spécifié. 10.1088/1751-8113/41/29/295204 . hal-03054036

\section{HAL Id: hal-03054036 \\ https://univ-angers.hal.science/hal-03054036}

Submitted on 11 Dec 2020

HAL is a multi-disciplinary open access archive for the deposit and dissemination of scientific research documents, whether they are published or not. The documents may come from teaching and research institutions in France or abroad, or from public or private research centers.
L'archive ouverte pluridisciplinaire HAL, est destinée au dépôt et à la diffusion de documents scientifiques de niveau recherche, publiés ou non, émanant des établissements d'enseignement et de recherche français ou étrangers, des laboratoires publics ou privés. 
The SOS model partition function and the elliptic weight functions

This content has been downloaded from IOPscience. Please scroll down to see the full text. 2008 J. Phys. A: Math. Theor. 41295204

(http://iopscience.iop.org/1751-8121/41/29/295204)

View the table of contents for this issue, or go to the journal homepage for more

Download details:

IP Address: 193.52.40.1

This content was downloaded on 17/07/2015 at 10:36

Please note that terms and conditions apply. 


\title{
The SOS model partition function and the elliptic weight functions"
}

\author{
S Pakuliak ${ }^{1,2}$, V Rubtsov ${ }^{2,3}$ and A Silantyev ${ }^{1,3}$ \\ ${ }^{1}$ Laboratory of Theoretical Physics, JINR, 141980 Dubna, Moscow reg., Russia \\ 2 Institute of Theoretical and Experimental Physics, Moscow 117259, Russia \\ ${ }^{3}$ Départment de Mathématiques, Université d’Angers, 2 Bd. Lavoisier, 49045 Angers, France \\ E-mail: pakuliak@theor.jinr.ru, Volodya.Roubtsov@univ-angers.fr and \\ silant@tonton.univ-angers.fr
}

Received 2 February 2008, in final form 3 June 2008

Published 26 June 2008

Online at stacks.iop.org/JPhysA/41/295204

\begin{abstract}
We generalized a recent observation (Khoroshkin and Pakuliak 2005 Theor. Math. Phys. 145 1373) that the partition function of the six-vertex model with domain wall boundary conditions can be obtained from a calculation of projections of the product of total currents in the quantum affine algebra $U_{q}\left(\widehat{\mathfrak{s l}}_{2}\right)$ in its current realization. A generalization is done for the elliptic current algebra (Enriquez and Felder 1998 Commun. Math. Phys. 195 651, Enriquez and Rubtsov 1997 Ann. Sci. Ecole Norm. Sup. 30 821). The projections of the product of total currents in this case are calculated explicitly and are presented as integral transforms of a product of the total currents. It is proved that the integral kernel of this transform is proportional to the partition function of the SOS model with domain wall boundary conditions.
\end{abstract}

PACS numbers: 02.20.-a, 02.20.Uw, 05.59.+q

\section{Introduction}

The main aim of this paper is to apply the elliptic current projection method to calculate the universal elliptic weight functions. The projections of currents first appeared in the works of Enriquez and the second author $[4,5]$. This was a method for constructing a higher genus analog of quantum groups in terms of Drinfeld currents [6]. The current (or 'new') realization supplies the quantum affine algebra with another co-product (the 'Drinfeld' co-product). The standard and Drinfeld co-products are related by a 'twist' (see [4]). The quantum algebra is decomposed (in two different ways) into a product of two 'Borel subalgebras'. We can consider (for each subalgebra) its intersection with two other Borel subalgebras and express it as their product. Thus we obtain for each subalgebra a pair of projection operators from

* This work is dedicated to the memory of Alexei Zamolodchikov. 
the subalgebra to each of these intersections. The above-mentioned twist is defined by a Hopf pairing of the subalgebras and the projection operators (see section 4 where we recall an elliptic version of this construction).

Further, Khoroshkin and the first author have applied this method for a factorization of the universal $R$-matrix [7] in the quantum affine algebras and to obtain a universal weight function [1, 8] for arbitrary quantum affine algebra. Most general elliptic weight functions were introduced by Tarasov and Varchenko in [11]. The weight functions play a fundamental role in the theory of deformed Knizhnik-Zamolodchikov and Knizhnik-Zamolodchikov-Bernard equations. In particular, in the case of $U_{q}\left(\widehat{\mathfrak{g l}}_{n}\right)$, acting by the projections of Drinfeld currents on the highest weight vectors of irreducible finite-dimensional representations, one obtains exactly the (trigonometric) weight functions or off-shell Bethe vectors. In the canonical nested Bethe ansatz these objects are defined implicitly by the recursive relations. Calculations of the projections are an effective way to resolve the hierarchical relations of the nested Bethe ansatz.

It was observed in [1] that the projections for the algebra $U_{q}\left(\widehat{\mathfrak{s l}_{2}}\right)$ can be presented as an integral transform and the integral kernel of this transform is proportional to the partition function of the finite six-vertex model with domain wall boundary conditions (DWBC) [1]. Here we prove that the elliptic projections described in [2] can help to derive the partition function for the elliptic models. It was shown in this paper that the calculation of the projections in the current elliptic algebra [2, 3] yields the partition function of the solid-on-solid (SOS) model with domain wall boundary conditions.

In [20] Korepin derived recurrent relations for the partition function for the finite six-vertex model with domain wall boundary conditions. Further Izergin used them to find the expression for the partition function in a determinant form [9]. The integral kernel of projections satisfies the same recursive relations and gives another formula for the partition function.

The problem of generalization of the Izergin's determinant formula to the elliptic case was extensively discussed in the last two decades. One can prove that the statistical sum of the SOS model with DWBC cannot be presented in the form of the single determinant. When this paper was prepared Rosengren [10] has shown that this statistical sum for $n \times n$ lattice can be written as a sum of $2^{n}$ determinants generalizing the Izergin's determinant formula. His approach relates to some (dynamical) generalization of alternating-sign matrices and goes along with the famous Kuperberg combinatorial demonstration [16].

We expect that the projection method gives a universal form of the elliptic weight function [11] as it does in the case of the quantum affine algebras [12]. When this universal weight function is presented as an integral transform of the product of the elliptic currents we show that the integral kernel of this transform gives an expression of the partition function for the SOS model. On one hand, we generalize Korepin's recurrent relations and on the other hand we generalize the method proposed in [1] for calculating the projections to the elliptic case. We check that the integral kernel extracted from the universal weight function and multiplied by a certain factor satisfies the obtained recurrent relations, which uniquely define the partition function for the SOS model with DWBC. Our formula given by the projection method coincides with the Rosengren's one.

An interesting open problem which still deserves more extensive studies is a relation of the projection method with elliptic Sklyanin-Odesskii-Feigin algebras. It was observed in the pioneering paper [3] that the half of elliptic current generators satisfies the commutation relations of $W$-elliptic algebras of Feigin. Another intriguing relation was observed in [17]: a certain subalgebra in the ' $\lambda$-generalization' of Sklyanin algebra (a graded algebra of meromorphic functions with ' $\lambda$-twisted' (anti)symmetrization product) satisfies the Felder $R$-matrix quadratic relations [18]. The latter paper gives a description of the elliptic Bethe 


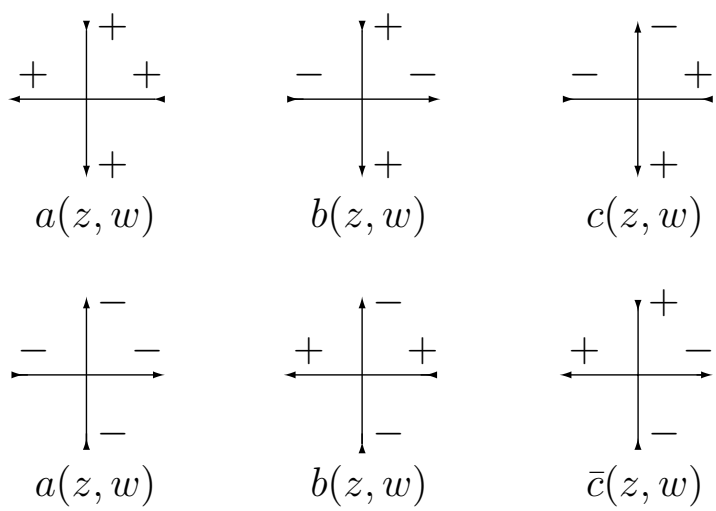

Figure 1. Graphical presentation of the Boltzmann weights.

eigenvectors or the elliptic weight functions. This is a strong indication that the projection method should be considered and interpreted in the framework of (generalized) SklyaninOdesskii-Feigin algebras. We hope to discuss it elsewhere.

The main results of the paper were reported in Seventh International Workshop 'Supersymmetry and Quantum Symmetry' in JINR, Dubna (Russia), 30 July-4 August, 2007.

The paper is organized as follows. In section 2, we briefly review the finite sixvertex model with DWBC and present the formulae for the partition function: the Izergin's determinant formula and the formula obtained by the projection method. Section 3 is devoted to the SOS model with DWBC. We introduce the model without great details and pose a problem how to calculate the partition function of this model. We obtain analytical properties of the partition function and prove that they allow us to reconstruct the partition function exactly. In section 4 , we introduce the projections in terms of the currents for the elliptic algebra following [2]. We generalize the method proposed in [1] to our case to obtain the integral representation of the projections of product of currents. Then, using a Hopf pairing we extract the integral kernel and check that it satisfies all necessary analytical properties of the partition function of the SOS model with DWBC. In section 5, we investigate a trigonometric limit of the elliptic model and the partition function with DWBC. We arrive at the six-vertex model case by two steps. The model obtained after the first step is a trigonometric SOS model. Then we show that the degeneration of the expression derived in section 4 coincides with the known expression for the six-vertex model partition function with DWBC. An appendix contains the necessary information on the properties of elliptic polynomials. This terminology goes back to Felder (see i.e. [13]). We should stress that our elliptic polynomial of order $n$ with a given character is also called by a 'theta-function of order $n$ norm $\alpha$ ' in [10].

\section{Partition function of the finite six-vertex model}

Let us consider a statistical system on a $n \times n$ square lattice, where the columns and rows are enumerated from 1 to $n$ from right to left and upward respectively. This is a six-vertex model where vertices on the lattice are associated with Boltzmann weights which depend on the configuration of the arrows around a given vertex. Six possible configurations are shown in figure 1 and weights are functions of two spectral parameters $z, w$ and anisotropy parameters $q$ :

$$
\begin{array}{ll}
a(z, w)=q z-q^{-1} w, & b(z, w)=z-w \\
c(z, w)=\left(q-q^{-1}\right) z, & \bar{c}(z, w)=\left(q-q^{-1}\right) w .
\end{array}
$$




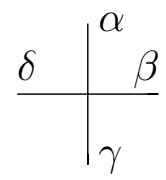

Figure 2. The Boltzmann weight $R(z, w)_{\gamma \delta}^{\alpha \beta}$.

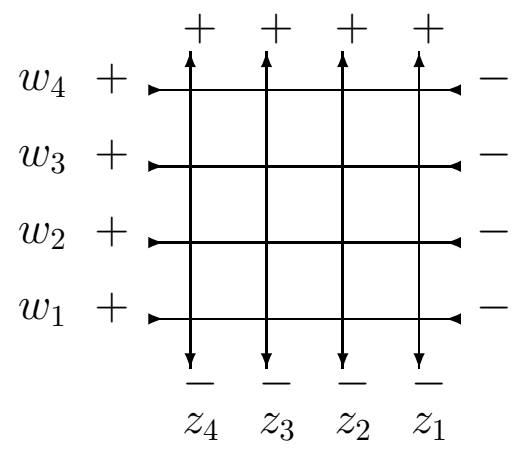

Figure 3. Inhomogeneous lattice with domain wall boundary conditions.

Let us associate the sign ' + ' with the upward and left directed arrows, while the sign '-' is associated with the downward and right directed arrows as shown in figure 1. The Boltzmann weights (1) are gathered in the matrix

$$
R(z, w)=\left(\begin{array}{cccc}
a(z, w) & 0 & 0 & 0 \\
0 & b(z, w) & \bar{c}(z, w) & 0 \\
0 & c(z, w) & b(z, w) & 0 \\
0 & 0 & 0 & a(z, w)
\end{array}\right)
$$

acting in the space $\mathbb{C}^{2} \otimes \mathbb{C}^{2}$ with the basis $e_{\alpha} \otimes e_{\beta}, \alpha, \beta= \pm$. The entry $R(z, w)_{\gamma \delta}^{\alpha \beta}, \alpha, \beta, \gamma, \delta=$ \pm coincides with the Boltzmann weight corresponding to figure 2:

Different repartitions of the arrows on the edges form different configurations $\{C\}$. A Boltzmann weight of the lattice is a product of the Boltzmann weights in each vertex. We define the partition function of the model summing the Boltzmann weights of the lattice over all possible configurations subject to some boundary conditions:

$$
Z(\{z\},\{w\})=\sum_{\{C\}} \prod_{i, j=1}^{n} R\left(z_{i}, w_{j}\right)_{\gamma_{i j} \delta_{i j}}^{\alpha_{i j} \beta_{i j}} .
$$

Here $\alpha_{i j}, \beta_{i j}, \gamma_{i j}, \delta_{i j}$ are corresponding signs around the $(i, j)$ th vertex. We consider an inhomogeneous model when the Boltzmann weights depend on the column via the variable $z_{i}$ and on the row via the variable $w_{j}$ (see figure 3).

We choose so-called domain wall boundary conditions (DWBC) that fix the boundary arrows (signs) as is shown in figure 3 . In other words, the arrows are entering on the left and right boundaries and leaving on the lower and upper ones. 
In [9], Izergin has found a determinant presentation for the partition function of the lattice with DWBC

$$
\begin{aligned}
Z(\{z\},\{w\})= & \left(q-q^{-1}\right)^{n} \prod_{m=1}^{n} w_{m} \frac{\prod_{i, j=1}^{n}\left(z_{i}-w_{j}\right)\left(q z_{i}-q^{-1} w_{j}\right)}{\prod_{n \geqslant i>j \geqslant 1}\left(z_{i}-z_{j}\right)\left(w_{j}-w_{i}\right)} \operatorname{det} \\
& \times\left\|\frac{1}{\left(z_{i}-w_{j}\right)\left(q z_{i}-q^{-1} w_{j}\right)}\right\|_{i, j=1, \ldots, n} \cdot
\end{aligned}
$$

The Izergin's idea was to use a symmetry of the polynomial (3) and Korepin's recurrent relations for the quantity $Z(\{z\},\{w\})$ to observe that these recurrent relations allow us to reconstruct $Z(\{z\},\{w\})$ in a unique way and that the same recurrent relations are valid for the determinant formula (4).

On the other hand, it was observed that the integral kernel of the projection of $n$ currents is a polynomial of the same degree and it satisfies the same Korepin's recurrent relations [1]. This means that this integral kernel coincides with the partition function for $n \times n$ lattice. Moreover, the theory of projections gives another expression for the partition function:

$$
\begin{aligned}
& Z(\{z\},\{w\})=\left(q-q^{-1}\right)^{n} \prod_{m=1}^{n} w_{m} \prod_{n \geqslant i>j \geqslant 1} \frac{q^{-1} w_{i}-q w_{j}}{w_{i}-w_{j}} \\
& \quad \times \sum_{\sigma \in S_{n}} \prod_{\substack{1 \leqslant i<j \leqslant n \\
\sigma(i)>\sigma(j)}} \frac{q w_{\sigma(i)}-q^{-1} w_{\sigma(j)}}{q^{-1} w_{\sigma(i)}-q w_{\sigma(j)}} \prod_{n \geqslant i>k \geqslant 1}\left(q z_{i}-q^{-1} w_{\sigma(k)}\right) \prod_{1 \leqslant i<k \leqslant n}\left(z_{i}-w_{\sigma(k)}\right),
\end{aligned}
$$

where $S_{n}$ is a permutation group. Here the factor $\frac{q w_{\sigma(i)}-q^{-1} w_{\sigma(j)}}{q^{-1} w_{\sigma(i)}-q w_{\sigma(j)}}$ appears in the product if both the conditions $i<j$ and $\sigma(i)>\sigma(j)$ are satisfied simultaneously. We have to remark that the representation (5) for the partition function of the six-vertex model with DWBC can be obtained directly from the coordinate Bethe ansatz.

\section{Partition function for the SOS model}

\subsection{Description of the SOS model}

The SOS model is a face model. We introduce it as, usually, in terms of heights, but then represent it in terms of $R$-matrix formalism as in [13]. This language is more convenient to generalize the results reviewed in section 2 and to prove the symmetry of the partition function.

Consider a square $n \times n$ lattice with the vertices enumerated by index $i=1, \ldots, n$ as in the previous case. It has $(n+1) \times(n+1)$ faces enumerated by pairs $(i, j), i, j=0, \ldots, n$ (see figure 4). To each face we put a complex number called height in such a way that the differences of the heights corresponding to the neighbor faces are \pm 1 . Let us denote by $d_{i j}$ the height corresponding to the face $(i, j)$ placed to the up-left from the vertex $(i, j)$. Then the last condition can be written in the form $\left|d_{i j}-d_{i-1, j}\right|=1$, for $i=1, \ldots, n, j=0, \ldots, n$, and $\left|d_{i j}-d_{i j-1}\right|=1$ for $i=0, \ldots, n, j=1, \ldots, n$. Each distribution of heights $d_{i j}$ $(i, j=0, \ldots, n)$ subject to these conditions and also to some boundary conditions defines a configuration of the model. This means that the partition function of this model can be presented in the form

$$
Z=\sum_{C} \prod_{i, j=1}^{n} W_{i j}\left(d_{i, j-1}, d_{i-1, j-1}, d_{i-1, j}, d_{i j}\right)
$$




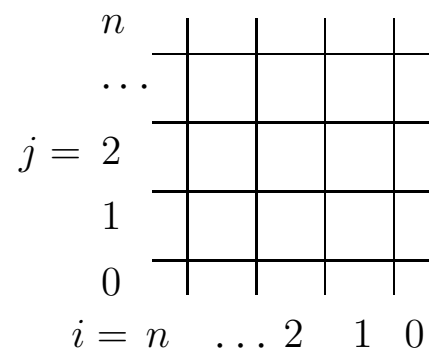

Figure 4. The numeration of faces.

where $W_{i j}\left(d_{i, j-1}, d_{i-1, j-1}, d_{i-1, j}, d_{i j}\right)$ are Boltzmann weights of $(i, j)$ th vertex depending on the configuration via connected heights as shown in figure 5 [14]:

$$
\begin{aligned}
& W_{i j}(d+1, d+2, d+1, d)=a\left(u_{i}-v_{j}\right)=\theta\left(u_{i}-v_{j}+\hbar\right), \\
& W_{i j}(d-1, d-2, d-1, d)=a\left(u_{i}-v_{j}\right)=\theta\left(u_{i}-v_{j}+\hbar\right), \\
& W_{i j}(d-1, d, d+1, d)=b\left(u_{i}-v_{j} ; \hbar d\right)=\frac{\theta\left(u_{i}-v_{j}\right) \theta(\hbar d+\hbar)}{\theta(\hbar d)}, \\
& W_{i j}(d+1, d, d-1, d)=\bar{b}\left(u_{i}-v_{j} ; \hbar d\right)=\frac{\theta\left(u_{i}-v_{j}\right) \theta(\hbar d-\hbar)}{\theta(\hbar d)}, \\
& W_{i j}(d-1, d, d-1, d)=c\left(u_{i}-v_{j} ; \hbar d\right)=\frac{\theta\left(u_{i}-v_{j}+\hbar d\right) \theta(\hbar)}{\theta(\hbar d)}, \\
& W_{i j}(d+1, d, d+1, d)=\bar{c}\left(u_{i}-v_{j} ; \hbar d\right)=\frac{\theta\left(u_{i}-v_{j}-\hbar d\right) \theta(\hbar)}{\theta(-\hbar d)} .
\end{aligned}
$$

As in the six-vertex case the variables $u_{i}, v_{j}$ are attached to the $i$ th vertical and $j$ th horizontal lines respectively, $\hbar$ is a nonzero anisotropy parameter ${ }^{4}$. The weights are expressed via the ordinary odd theta-function defined by the conditions

$\theta(u+1)=-\theta(u), \quad \theta(u+\tau)=-e^{-2 \pi i u-\pi i \tau} \theta(u), \quad \theta^{\prime}(0)=1$.

Let us introduce the notations

$$
\begin{array}{ll}
\alpha_{i j}=d_{i-1, j}-d_{i j}, & \beta_{i j}=d_{i-1, j-1}-d_{i-1, j}, \\
\gamma_{i j}=d_{i-1, j-1}-d_{i, j-1}, & \delta_{i j}=d_{i, j-1}-d_{i j} .
\end{array}
$$

The differences (9) take the values \pm 1 and we attach them to the corresponding edges as in figure 2: $\gamma_{i, j+1}=\alpha_{i j}$ is the sign attached to the vertical edge connecting the $(i, j)$ th vertex with the $(i, j+1)$ st one, $\beta_{i, j+1}=\delta_{i j}$ is the sign attached to the horizontal edge connecting the $(i, j)$ th vertex with the $(i+1, j)$ th one. The configuration can be considered as a distribution of these signs on the internal edges which are subjected to the conditions $\alpha_{i j}+\beta_{i j}=\gamma_{i j}+\delta_{i j}, i, j=1, \ldots, n$. In terms of signs on external edges the DWBC are the same as shown in figure 3. Additionally, we have to fix one of the boundary heights, for example, $d_{n n}$. 4 In the elliptic case we use additive variables $u_{i}, v_{j}$ and an additive anisotropy parameter $\hbar$ instead of the
multiplicative variables $z_{i}=\mathrm{e}^{2 \pi \mathrm{i} u_{i}}, w_{i}=\mathrm{e}^{2 \pi \mathrm{i} v_{i}}$ and the multiplicative parameter $q=\mathrm{e}^{\pi \mathrm{i} \hbar}$. 


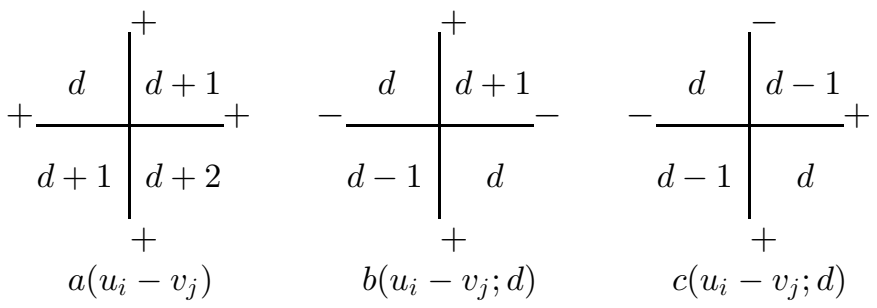

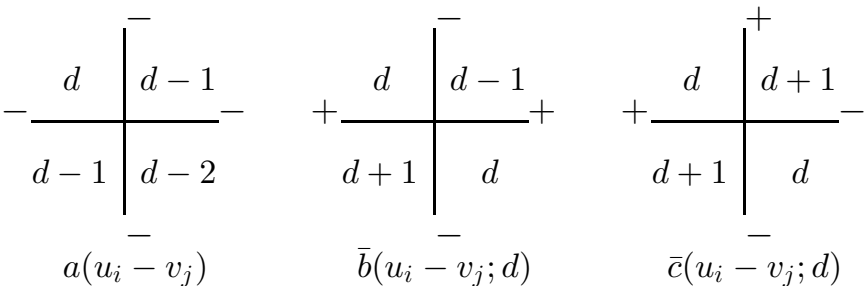

Figure 5. The Boltzmann weights for the SOS model.

The Boltzmann weights (7) can be presented as entries of the dynamical elliptic $R$-matrix [13]:

$$
\begin{aligned}
& W_{i j}\left(d_{i, j-1}, d_{i-1, j-1}, d_{i-1, j}, d_{i j}\right)=R\left(u_{i}-v_{j} ; \hbar d_{i j}\right)_{\gamma_{i j} \delta_{i j}}^{\alpha_{i j} \beta_{i j}}, \\
& R(u ; \lambda)=\left(\begin{array}{cccc}
a(u) & 0 & 0 & 0 \\
0 & b(u ; \lambda) & \bar{c}(u ; \lambda) & 0 \\
0 & c(u ; \lambda) & \bar{b}(u ; \lambda) & 0 \\
0 & 0 & 0 & a(u)
\end{array}\right) .
\end{aligned}
$$

Let $\mathbb{T}_{\gamma_{i 1}}^{\alpha_{i n}}\left(u_{i},\{v\}, \lambda_{i}\right)$ be shape column transfer matrices. It is a matrix-valued function of $u_{i}$, all spectral parameters $v_{j}, j=1, \ldots, n$ and the parameters $\lambda_{i}$ related to the heights:

$\mathbb{T}_{\gamma_{i 1}}^{\alpha_{i n}}\left(u_{i},\{v\}, \lambda_{i}\right)_{\delta_{i n} \ldots \delta_{i 1}}^{\beta_{i n} \ldots \beta_{i 1}}$

$=\left(R^{(n+1, n)}\left(u_{i}-v_{n} ; \lambda_{i n}\right) R^{(n+1, n-1)}\left(u_{i}-v_{n-1} ; \lambda_{i, n-1}\right) \cdots R^{(n+1,1)}\left(u_{i}-v_{1} ; \lambda_{i 1}\right)\right)_{\gamma_{i 1} ; \delta_{i n} \ldots \delta_{i 1}}^{\alpha_{i n} ; \beta_{i n} \ldots \beta_{i 1}}$

$=\left(R^{(n+1, n)}\left(u_{i}-v_{n} ; \Lambda_{i n}\right) R^{(n+1, n-1)}\left(u_{i}-v_{n-1} ; \Lambda_{i, n-1}\right) \cdots R^{(n+1,1)}\left(u_{i}-v_{1} ; \Lambda_{i 1}\right)\right)_{\gamma_{i 1} ; \delta_{i n} \ldots \delta_{i 1}}^{\alpha_{i n} ; \beta_{i n} \ldots \beta_{i 1}}$,

where $\lambda_{i j}=\hbar d_{i j}=\lambda_{i}+\hbar \sum_{l=j+1}^{n} \delta_{i l}, \lambda_{i}=\hbar d_{i n}=\lambda+\hbar \sum_{l=i+1}^{n} \alpha_{l n}, \Lambda_{i j}=\lambda_{i}+\hbar \sum_{l=j+1}^{n} H^{(l)}$. The matrix $H^{(l)}$ acts in the $l$ th two-dimensional space $V_{l} \cong \mathbb{C}^{2}$ as $\operatorname{diag}(1,-1)$ and $R$-matrix $R^{(a, b)}$ acts nontrivially in the tensor product of $V_{a} \otimes V_{b}$. The superscript $n+1$ in the $R$-matrices is regarded as an auxiliary space $V_{n+1} \cong \mathbb{C}^{2}$. The partition function (6) corresponding to DWBC $\left(\alpha_{i n}=+1, \beta_{1 i}=-1, \gamma_{i 1}=-1, \delta_{n i}=+1, i=1, \ldots, n\right)$ can be represented through the column transfer matrices:

$$
Z_{-+}^{+-}(\{u\},\{v\} ; \lambda)=\left(\mathbb{T}_{-}^{+}\left(u_{1},\{v\}, \lambda_{1}\right) \cdots \mathbb{T}_{-}^{+}\left(u_{n},\{v\}, \lambda_{n}\right)\right)_{+\ldots+}^{-\ldots-},
$$

where $\lambda_{i}=\lambda+\hbar(n-i)$. Similarly one can define the row transfer matrix.

\subsection{Analytical properties of the partition function}

Here we describe the analytical properties of the SOS model partition function analogical to those used by Izergin to restore the partition function of the six-vertex model. These properties uniquely define this partition function. 
Proposition 1. The partition function with $D W B C Z_{-+}^{+-}(\{u\},\{v\} ; \lambda)$ is a symmetric function in both sets of the variables $u_{i}$ and $v_{j}$.

Proof. Proof is based on the dynamical Yang-Baxter equation (DYBE) for the $R$-matrix [13] $R^{(12)}\left(t_{1}-t_{2} ; \lambda\right) R^{(13)}\left(t_{1}-t_{3} ; \lambda+\hbar H^{(2)}\right) R^{(23)}\left(t_{2}-t_{3} ; \lambda\right)$

$$
=R^{(23)}\left(t_{2}-t_{3} ; \lambda+\hbar H^{(1)}\right) R^{(13)}\left(t_{1}-t_{3} ; \lambda\right) R^{(12)}\left(t_{1}-t_{2} ; \lambda+\hbar H^{(3)}\right) .
$$

In order to prove the symmetry of partition function $Z_{-+}^{+-}(\{u\},\{v\} ; \lambda)$ under permutation $v_{j} \leftrightarrow v_{j-1}$ we rewrite DYBE in the form

$$
\begin{aligned}
R^{(n+1, j)}\left(u_{i}-v_{j}\right. & \left.; \Lambda_{i j}\right) R^{(n+1, j-1)}\left(u_{i}-v_{j-1} ; \Lambda_{i j}+\hbar H^{(j)}\right) \\
& \times R^{(j, j-1)}\left(v_{j}-v_{j-1} ; \Lambda_{i j}\right)=R^{(j, j-1)}\left(v_{j}-v_{j-1} ; \Lambda_{i j}+\hbar H^{(n+1)}\right) \\
& \times R^{(n+1, j-1)}\left(u_{i}-v_{j-1} ; \Lambda_{i j}\right) R^{(n+1, j)}\left(u_{i}-v_{j} ; \Lambda_{i j}+\hbar H^{(j-1)}\right) .
\end{aligned}
$$

Multiplying the $i$ th column matrix (11) by $R^{(j, j-1)}\left(v_{j}-v_{j-1} ; \Lambda_{i j}\right)$ from the right and moving this $R$-matrix to the left using (13), the relation $\left[H_{1}+H_{2}, R(u, \lambda)\right]=0$ and the equality $\Lambda_{i j}+\hbar \alpha_{i n}=\Lambda_{i-1, j}$ we obtain

$$
\begin{aligned}
\mathbb{T}_{\gamma_{i 1}}^{\alpha_{i n}}\left(u_{i},\{v\}, \lambda_{i}\right) & R^{(j, j-1)}\left(v_{j}-v_{j-1} ; \Lambda_{i j}\right)=R^{(j, j-1)}\left(v_{j}-v_{j-1} ; \Lambda_{i-1, j}\right) \\
\times & \mathcal{P}^{(j, j-1)} \mathbb{T}_{\gamma_{i 1}}^{\alpha_{i n}}\left(u_{i},\left\{v_{j} \leftrightarrow v_{j-1}\right\}, \lambda_{i}\right) \cdots \mathbb{T}_{\gamma_{n 1}}^{\alpha_{n n}}\left(u_{n},\left\{v_{j} \leftrightarrow v_{j-1}\right\}, \lambda_{n}\right) \mathcal{P}^{(j, j-1)},
\end{aligned}
$$

where $\mathcal{P} \in \operatorname{End}\left(\mathbb{C}^{2} \otimes \mathbb{C}^{2}\right)$ is a permutation matrix: $\mathcal{P}\left(e_{1} \otimes e_{2}\right)=e_{2} \otimes e_{1}$ for all $e_{1}, e_{2} \in \mathbb{C}^{2}$ and notation $\left\{v_{j} \leftrightarrow v_{j-1}\right\}$ means the set of the parameters $\{v\}$ with $v_{j-1}$ and $v_{j}$ are interchanged. Multiplying the product of the column matrix by $R^{(j, j-1)}\left(v_{j}-v_{j-1} ; \Lambda_{n j}\right)$ from right and moving it to the left using (14) one yields

$$
\begin{array}{r}
\mathbb{T}_{\gamma_{11}}^{\alpha_{1 n}}\left(u_{1},\{v\}, \lambda_{1}\right) \cdots \mathbb{T}_{\gamma_{n 1}}^{\alpha_{n n}}\left(u_{n},\{v\}, \lambda_{n}\right) R^{(j, j-1)}\left(v_{j}-v_{j-1} ; \Lambda_{n j}\right)=R^{(j, j-1)}\left(v_{j}-v_{j-1} ; \Lambda_{0, j}\right) \\
\times \mathcal{P}^{(j, j-1)} \mathbb{T}_{\gamma_{11}}^{\alpha_{1 n}}\left(u_{1},\left\{v_{j} \leftrightarrow v_{j-1}\right\}, \lambda_{1}\right) \cdots \mathbb{T}_{\gamma_{n 1}}^{\alpha_{n n}}\left(u_{n},\left\{v_{j} \leftrightarrow v_{j-1}\right\}, \lambda_{n}\right) \mathcal{P}^{(j, j-1)},
\end{array}
$$

where $\Lambda_{0, j}=\lambda+\hbar \sum_{i=1}^{n} \alpha_{i n}+\hbar \sum_{l=j+1}^{n} H^{(l)}, \Lambda_{n j}=\lambda_{n}=\lambda$. Eventually, comparing the matrix element $(\cdot)_{+, \ldots,+}^{-, \ldots,-}$ of both hand sides of (15), taking into account formula (12) and the identities

$$
\begin{aligned}
& R(u, \lambda)_{\gamma \delta}^{--}=a(u) \delta_{\gamma}^{-} \delta_{\delta}^{-}, \quad R(u, \lambda)_{++}^{\alpha \beta}=a(u) \delta_{+}^{\alpha} \delta_{+}^{\beta}, \\
& \mathcal{P}_{\gamma \delta}^{--}=\delta_{\gamma}^{-} \delta_{\delta}^{-}, \quad \mathcal{P}_{++}^{\alpha \beta}=\delta_{+}^{\alpha} \delta_{+}^{\beta},
\end{aligned}
$$

(where $\delta_{\gamma}^{\alpha}$ is a Kronecker's symbol) and substituting $\alpha_{i n}=+1, \gamma_{i 1}=-1$ one derives

$$
Z_{-+}^{+-}(\{u\},\{v\} ; \lambda)=Z_{-+}^{+-}\left(\{u\},\left\{v_{j} \leftrightarrow v_{j-1}\right\} ; \lambda\right) .
$$

Similarly, using the row transfer matrix one can obtain the following equality from DYBE:

$$
Z_{-+}^{+-}(\{u\},\{v\} ; \lambda)=Z_{-+}^{+-}\left(\left\{u_{j} \leftrightarrow u_{j-1}\right\},\{v\} ; \lambda\right) .
$$

The partition function with DWBC satisfies relations (16) and (17) for each $j=1, \ldots, n$, which is sufficient to establish the symmetry under an arbitrary permutation.

Proposition 2. The partition function with DWBC (12) is an elliptic polynomial ${ }^{5}$ of degree $n$ with the character $\chi$ in each variable $u_{i}$, where

$$
\chi(1)=(-1)^{n}, \quad \chi(\tau)=(-1)^{n} \exp \left(2 \pi \mathrm{i}\left(\lambda+\sum_{j=1}^{n} v_{j}\right)\right)
$$

5 The notion of elliptic polynomials and their properties are given in appendix A. 
Due to the symmetry with respect to the variables $\{u\}$ it is sufficient to prove the proposition for variable $u_{n}$. To present explicitly a dependence of $Z_{-+}^{+-}(\{u\},\{v\} ; \lambda)$ on $u_{n}$ we consider all possibilities for states of edges attached to the vertices located in the $n$th column. First consider the $(n, n)$ th vertex. Due to the boundary conditions $\alpha_{n n}=\delta_{n n}=+1$ and to the condition $\alpha_{n n}+\beta_{n n}=\gamma_{n n}+\delta_{n n}$ we have two possibilities: either $\beta_{n n}=\gamma_{n n}=-1$ or $\beta_{n n}=\gamma_{n n}=+1$. In the first case one has a unique possibility for the whole residual part of $n$th column: $\gamma_{n j}=-1, \beta_{n j}=+1, j=1, \ldots, n-1$; in the second case there are two possibilities for the $(n, n-1)$ st vertex: either $\beta_{n, n-1}=\gamma_{n, n-1}=-1$ or $\beta_{n, n-1}=\gamma_{n, n-1}=+1$, etc. Finally the partition function is represented in the form

$$
\begin{aligned}
Z_{-+}^{+-}(\{u\},\{v\} ; \lambda) & =\sum_{k=1}^{n} \prod_{j=k+1}^{n} a\left(u_{n}-v_{j}\right) \bar{c}\left(u_{n}-v_{k} ; \lambda+(n-k) \hbar\right) \\
& \times \prod_{j=1}^{k-1} \bar{b}\left(u_{n}-v_{j} ; \lambda+(n-j) \hbar\right) g_{k}\left(u_{n-1}, \ldots, u_{1},\{v\} ; \lambda\right),
\end{aligned}
$$

where $g_{k}\left(u_{n-1}, \ldots, u_{1},\{v\} ; \lambda\right)$ are functions not depending on $u_{n}$. Each term of this sum is an elliptic polynomial of degree $n$ with the same character (18) in the variable $u_{n}$.

Remark 1. Similarly one can prove that the function $Z_{-+}^{+-}(\{u\},\{v\} ; \lambda)$ is an elliptic polynomial of degree $n$ with the character $\tilde{\chi}$ in each variable $v_{i}$, where $\tilde{\chi}(1)=(-1)^{n}$, $\tilde{\chi}(\tau)=(-1)^{n} \mathrm{e}^{2 \pi \mathrm{i}\left(-\lambda+\sum_{i=1}^{n} u_{i}\right)}$.

Proposition 3. The nth partition function with DWBC (12) restricted to the condition $u_{n}=v_{n}-\hbar$ can be expressed through the $(n-1)$ st partition function:

$$
\begin{aligned}
Z_{-+}^{+-}\left(u_{n}=v_{n}-\right. & \left.\hbar, u_{n-1}, \ldots, u_{1} ; v_{n}, v_{n-1}, \ldots, v_{1} ; \lambda\right)=\frac{\theta(\lambda+n \hbar) \theta(\hbar)}{\theta(\lambda+(n-1) \hbar)} \\
& \times \prod_{m=1}^{n-1}\left(\theta\left(v_{n}-v_{m}-\hbar\right) \theta\left(u_{m}-v_{n}\right)\right) Z_{-+}^{+-}\left(u_{n-1}, \ldots, u_{1} ; v_{n-1}, \ldots, v_{1} ; \lambda\right) .
\end{aligned}
$$

Considering the $n$th column and the $n$th row and taking into account that $\left.a\left(u_{n}-v_{n}\right)\right|_{u_{n}=v_{n}-\hbar}=$ $a(-\hbar)=0$ we conclude that the unique possibility of having a non-trivial contribution is $\beta_{n n}=\gamma_{n n}=-1, \gamma_{n j}=-1, \beta_{n j}=+1, j=1, \ldots, n-1, \beta_{i n}=-1, \gamma_{i n}=+1, i=$ $1, \ldots, n-1$. The last formulae impose the same DWBC for the $(n-1) \times(n-1)$ sublattice: $\delta_{n-1, j}=\beta_{n j}=+1, j=1, \ldots, n-1, \alpha_{i, n-1}=\gamma_{i n}=+1, i=1, \ldots, n-1, d_{n-1, n-1}=d_{n n}$. Thus the substitution $u_{n}=v_{n}-\hbar$ into the partition function for the whole lattice gives us

$$
\begin{aligned}
Z_{-+}^{+-}\left(u_{n}=v_{n}-\right. & \left.\hbar, u_{n-1}, \ldots, u_{1} ; v_{n}, v_{n-1}, \ldots, v_{1} ; \lambda\right) \\
= & \bar{c}(-\hbar ; \lambda) \prod_{j=1}^{n-1} \bar{b}\left(v_{n}-v_{j}-\hbar ; \lambda+(n-j) \hbar\right) \prod_{i=1}^{n-1} b\left(u_{i}-v_{n} ; \lambda+(n-\mathrm{i}) \hbar\right) \\
& \times Z_{-+}^{+-}\left(u_{n-1}, \ldots, u_{1} ; v_{n-1}, \ldots, v_{1} ; \lambda\right) .
\end{aligned}
$$

Using the explicit expressions for the Boltzmann weights (7) one can rewrite the last formula in the form (19).

Remark 2. From formula (20) we see that the following transformation of the $R$-matrix,

$$
b(u, v ; \lambda) \rightarrow \rho b(u, v ; \lambda), \quad \bar{b}(u, v ; \lambda) \rightarrow \rho^{-1} \bar{b}(u, v ; \lambda)
$$

does not change the recurrent relation (19), where $\rho$ is a non-zero constant not depending on $u, v$ and $\lambda$. 
Lemma 1. If the set of functions $\left\{Z^{(n)}\left(u_{n}, \ldots, u_{1} ; v_{n}, \ldots, v_{1} ; \lambda\right)\right\}_{n \geqslant 1}$ satisfies the conditions of the propositions 1, 2, 3 and the initial condition

$$
Z^{(1)}\left(u_{1} ; v_{1} ; \lambda\right)=\bar{c}\left(u_{1}-v_{1}\right)=\frac{\theta\left(u_{1}-v_{1}-\lambda\right) \theta(\hbar)}{\theta(-\lambda)}
$$

then

$$
Z_{-+}^{+-}\left(u_{n}, \ldots, u_{1} ; v_{n}, \ldots, v_{1} ; \lambda\right)=Z^{(n)}\left(u_{n}, \ldots, u_{1} ; v_{n}, \ldots, v_{1} ; \lambda\right)
$$

This lemma can be proved by induction over $n$. Let the equality (23) be valid for $n-1$. Consider the functions $Z_{-+}^{+-}\left(u_{n}, \ldots, u_{1} ; v_{n}, \ldots, v_{1} ; \lambda\right)$ and $Z^{(n)}\left(u_{n}, \ldots, u_{1} ; v_{n}, \ldots, v_{1} ; \lambda\right)$ as functions of $u_{n}$. Both are elliptic polynomials of degree $n$ with the character (18). They have the same value in the point $u_{n}=v_{n}-\hbar$, and due to the symmetry of these functions with respect to the parameters $\left\{v_{j}\right\}_{j=1}^{n}$ they coincide in all the points $u_{n}=v_{j}-\hbar, j=1, \ldots, n$. Then, from lemma 2 (see appendix A) these functions are identical.

Remark 3. As we see from the proof of lemma 1 it is sufficient to establish the symmetry with respect to only the variables $v_{j}$.

Remark 4. The transformation (21) of the $R$-matrix does not change the partition function with DWBC.

\section{Elliptic projections of currents}

Let $\mathcal{K}_{0}=\mathbb{C}\left[u^{-1}\right][[u]]$ be a complete set of complex-valued meromorphic functions defined in the neighborhood of origin, which have only simple poles at this point. Let $\left\{\epsilon^{i}\right\}$ and $\left\{\epsilon_{i}\right\}$ be two dual bases in $\mathcal{K}_{0} \oint \frac{\mathrm{d} u}{2 \pi \mathrm{i}} \epsilon^{i}(u) \epsilon_{j}(u)=\delta_{j}^{i}$.

\subsection{Current description of the elliptic algebra}

Let $\mathcal{A}$ be a Hopf algebra generated by elements $\hat{h}[s], \hat{e}[s], \hat{f}[s], s \in \mathcal{K}_{0}$, which are subjected to linear relations

$$
\hat{x}\left[\alpha_{1} s_{1}+\alpha_{2} s_{2}\right]=\alpha_{1} \hat{x}\left[s_{1}\right]+\alpha_{2} \hat{x}\left[s_{2}\right], \quad \alpha_{1}, \alpha_{2} \in \mathbb{C}, \quad s_{1}, s_{2} \in \mathcal{K}_{0},
$$

where $x \in\{h, e, f\}$ and some commutation relations. These relations will be written in terms of currents

$$
\begin{aligned}
h^{+}(u) & =\sum_{i \geqslant 0} \hat{h}\left[\epsilon_{i ; 0}\right] \epsilon^{i ; 0}(u), & & h^{-}(u)=-\sum_{i \geqslant 0} \hat{h}\left[\epsilon^{i ; 0}\right] \epsilon_{i ; 0}(u), \\
f(u) & =\sum_{i} \hat{f}\left[\epsilon_{i}\right] \epsilon^{i}(u), & e(u) & =\sum_{i} \hat{e}\left[\epsilon_{i}\right] \epsilon^{i}(u) .
\end{aligned}
$$

The currents $e(u)$ and $f(u)$ are called total currents. They are defined by dual bases of $\mathcal{K}_{0}$ and their definition does not depend on the choice of these dual bases (see [3, 15]). The currents $h^{+}(u)$ and $h^{-}(u)$ are called Cartan currents and they are defined by a special basis

$$
\epsilon^{k ; 0}(u)=\frac{1}{k !}\left(\frac{\theta^{\prime}(u)}{\theta(u)}\right)^{(k)}, \quad k \geqslant 0 ; \quad \epsilon_{k ; 0}(u)=(-u)^{k}, \quad k \geqslant 0 .
$$

The commutation relations read as follows [2]:

$$
\left[K^{ \pm}(u), K^{ \pm}(v)\right]=0, \quad\left[K^{+}(u), K^{-}(v)\right]=0,
$$




$$
\begin{aligned}
& K^{ \pm}(u) e(v) K^{ \pm}(u)^{-1}=\frac{\theta(u-v+\hbar)}{\theta(u-v-\hbar)} e(v), \\
& K^{ \pm}(u) f(v) K^{ \pm}(u)^{-1}=\frac{\theta(u-v-\hbar)}{\theta(u-v+\hbar)} f(v), \\
& \theta(u-v-\hbar) e(u) e(v)=\theta(u-v+\hbar) e(v) e(u), \\
& \theta(u-v+\hbar) f(u) f(v)=\theta(u-v-\hbar) f(v) f(u), \\
& {[e(u), f(v)]=\hbar^{-1} \delta(u, v)\left(K^{+}(u)-K^{-}(v)\right),}
\end{aligned}
$$

where $K^{+}(u)=\exp \left(\frac{\mathrm{e}^{\hbar \partial_{u}}-\mathrm{e}^{-\hbar \partial_{u}}}{2 \partial_{u}} h^{+}(u)\right), K^{-}(u)=\exp \left(\hbar h^{-}(u)\right)$ and $\delta(u, v)=\sum_{n \in \mathbb{Z}} \frac{u^{n}}{v^{n+1}}$ is a delta-function ${ }^{6}$ for $\mathcal{K}_{0}$. The algebra $\mathcal{A}$ is a non-central version of the algebra $A(\tau)$ introduced in [3]. This algebra is equipped with the co-product and co-unit:

$$
\begin{aligned}
& \Delta K^{ \pm}(u)=K^{ \pm}(u) \otimes K^{ \pm}(u), \\
& \Delta e(u)=e(u) \otimes 1+K^{-}(u) \otimes e(u), \\
& \Delta f(u)=f(u) \otimes K^{+}(u)+1 \otimes f(u), \\
& \varepsilon\left(K^{ \pm}(u)\right)=1, \quad \varepsilon(e(u))=0, \quad \varepsilon(f(u))=0 .
\end{aligned}
$$

Let $\mathcal{A}_{F}$ and $\mathcal{A}_{E}$ be subalgebras of $\mathcal{A}$ generated by the generators $\hat{h}\left[\epsilon_{i ; 0}\right], \hat{f}[s]$, and $\hat{h}\left[\epsilon^{i ; 0}\right], \hat{e}[s]$, respectively, $s \in \mathcal{K}_{0}$. The subalgebra $\mathcal{A}_{F}$ is described by currents $K^{+}(u), f(u)$, and the subalgebra $\mathcal{A}_{E}$ by $K^{-}(u), e(u)$. Also, we introduce notations $H^{+}$for the subalgebras of $\mathcal{A}$ generated by $\hat{h}\left[\epsilon_{i ; 0}\right]$. As was stated in [2] the bialgebras $\left(\mathcal{A}_{F}, \Delta^{o p}\right)$ and $\left(\mathcal{A}_{E}, \Delta\right)$ are dual to each other with respect to the Hopf pairing $\langle\cdot, \cdot\rangle: \mathcal{A}_{F} \times \mathcal{A}_{E} \rightarrow \mathbb{C}$ defined in terms of currents as follows: $\left\langle f(u), K^{-}(v)\right\rangle=\left\langle K^{+}(u), e(v)\right\rangle=0$ and

$$
\langle f(u), e(v)\rangle=\hbar^{-1} \delta(u, v), \quad\left\langle K^{+}(u), K^{-}(v)\right\rangle=\frac{\theta(u-v-\hbar)}{\theta(u-v+\hbar)} .
$$

These formulae uniquely define the Hopf pairing on $\mathcal{A}_{F} \times \mathcal{A}_{E}$. In particular, one can derive the following formula (see appendix B):

$$
\left\langle f\left(t_{n}\right) \cdots f\left(t_{1}\right), e\left(v_{n}\right) \cdots e\left(v_{1}\right)\right\rangle=\hbar^{-n} \sum_{\sigma \in S_{n}} \prod_{\substack{l<l^{\prime} \\ \sigma(l)>\sigma\left(l^{\prime}\right)}} \frac{\theta\left(v_{\sigma(l)}-v_{\sigma\left(l^{\prime}\right)}+\hbar\right)}{\theta\left(v_{\sigma(l)}-v_{\sigma\left(l^{\prime}\right)}-\hbar\right)} \prod_{m=1}^{n} \delta\left(t_{m}, v_{\sigma(m)}\right)
$$

\subsection{Projections of currents}

We define the projections as linear maps acting in the subalgebra $\mathcal{A}_{F}$. In the subalgebra $\mathcal{A}_{E}$ dual projections act, which we do not consider here. The main tools to work with the projections are shape half-currents $f_{\lambda}^{+}(u)$ and $f_{\lambda}^{-}(u)$ (defined further). For this reason we define the projections in terms of the half-currents. The last ones are usually defined as parts of the sum (24) (with the corresponding sign) such that $f(u)=f_{\lambda}^{+}(u)-f_{\lambda}^{-}(u)$. Here $\lambda$ is a parameter of the decomposition of the total current into the difference of half-currents.

6 One can find more details about distributions acting on $\mathcal{K}_{0}$ and their significance in the theory of current algebras in our previous paper [15]. 
Elliptic half-currents are investigated in detail on the classical level in [15]. We will introduce the half-currents by their representations via integral transforms of the total current $f(u)$ :

$$
\begin{aligned}
f_{\lambda}^{+}(u) & =\oint_{|v|<|u|} \frac{\mathrm{d} v}{2 \pi \mathrm{i}} \frac{\theta(u-v-\lambda)}{\theta(u-v) \theta(-\lambda)} f(v), \\
f_{\lambda}^{-}(u) & =\oint_{|v|>|u|} \frac{\mathrm{d} v}{2 \pi \mathrm{i}} \frac{\theta(u-v-\lambda)}{\theta(u-v) \theta(-\lambda)} f(v),
\end{aligned}
$$

where $\lambda \notin \Gamma=\mathbb{Z}+\mathbb{Z} \tau$. The half-current $f_{\lambda}^{+}(u)$ is called positive and $f_{\lambda}^{-}(u)$-negative.

The corresponding positive and negative projections are also parameterized by $\lambda$ and they are defined on the half-currents as follows:

$$
\begin{array}{rrr}
P_{\lambda}^{+}\left(f_{\lambda}^{+}(u)\right)=f_{\lambda}^{+}(u), & P_{\lambda}^{-}\left(f_{\lambda}^{+}(u)\right)=0, \\
P_{\lambda}^{+}\left(f_{\lambda}^{-}(u)\right)=0, & P_{\lambda}^{-}\left(f_{\lambda}^{-}(u)\right)=f_{\lambda}^{-}(u) .
\end{array}
$$

Let us first define the projections in subalgebra $\mathcal{A}_{f}$ generated by currents $f(u)$. As a linear space this subalgebra is spanned by the products $f\left(u_{n}\right) f\left(u_{n-1}\right) \cdots f\left(u_{1}\right), n=0,1,2, \ldots$ This means that any element of $\mathcal{A}_{f}$ can be presented as a sum (maybe infinite) of the integrals ${ }^{7}$

$$
\oint \frac{\mathrm{d} u_{n} \cdots \mathrm{d} u_{1}}{(2 \pi i)^{n}} f\left(u_{n}\right) \cdots f\left(u_{1}\right) s_{n}\left(u_{n}\right) \cdots s_{1}\left(u_{1}\right), \quad s_{n}, \ldots, s_{1} \in \mathcal{K}_{0} .
$$

It follows from the PBW theorem proved in [2] that any element of $\mathcal{A}_{f}$ can also be represented as a sum of the integrals

$\oint \frac{\mathrm{d} u_{n} \cdots \mathrm{d} u_{1}}{(2 \pi \mathrm{i})^{n}} f_{\lambda+2(n-1) \hbar}^{-}\left(u_{n}\right) \cdots f_{\lambda+2 m \hbar}^{-}\left(u_{m+1}\right) f_{\lambda+2(m-1) \hbar}^{+}\left(u_{m}\right) \cdots f_{\lambda}^{+}\left(u_{1}\right) s_{n}\left(u_{n}\right) \cdots s_{1}\left(u_{1}\right)$,

$s_{n} \cdots s_{1} \in \mathcal{K}_{0}, 0 \leqslant m \leqslant n$, and, therefore, it is sufficient to define the projections on these products of half-currents:

$$
P_{\lambda}^{+}\left(x^{-} x^{+}\right)=\varepsilon\left(x^{-}\right) x^{+}, \quad P_{\lambda}^{-}\left(y^{-} y^{+}\right)=y^{-} \varepsilon\left(y^{+}\right),
$$

where

$$
\begin{array}{ll}
x^{-}=f_{\lambda+2(n-1) \hbar}^{-}\left(u_{n}\right) \cdots f_{\lambda+2 m \hbar}^{-}\left(u_{m+1}\right), & y^{-}=f_{\lambda}^{-}\left(u_{n}\right) \cdots f_{\lambda-2(n-m-1) \hbar}^{-}\left(u_{m+1}\right), \\
x^{+}=f_{\lambda+2(m-1) \hbar}^{+}\left(u_{m}\right) \cdots f_{\lambda}^{+}\left(u_{1}\right), & y^{+}=f_{\lambda-2(n-m) \hbar}^{+}\left(u_{m}\right) \cdots f_{\lambda-2(n-1) \hbar}^{+}\left(u_{1}\right) .
\end{array}
$$

The product of zero number of currents is identified with 1 and in this case: $\varepsilon(1)=1$. The counit $\varepsilon$ of nonzero number of half-currents is always zero. So, this definition generalizes formulae (38) and (39). We complete the definition of the projections on all the subalgebras $\mathcal{A}_{F}=\mathcal{A}_{f} \cdot H^{+}$by formulae

$$
P_{\lambda}^{+}\left(a t^{+}\right)=P_{\lambda}^{+}(a) t^{+}, \quad P_{\lambda}^{-}\left(a t^{+}\right)=P_{\lambda}^{-}(a) \varepsilon\left(t^{+}\right),
$$

where $a \in \mathcal{A}_{f}, t^{+} \in H^{+}$.

\subsection{The projections and the universal elliptic weight function}

Consider the expressions of the form

$$
P_{\lambda-(n-1) \hbar}^{+}\left(f\left(u_{n}\right) f\left(u_{n-1}\right) \cdots f\left(u_{2}\right) f\left(u_{1}\right)\right),
$$

where the parameter $\lambda-(n-1) \hbar$ is chosen for some symmetry reasons. Let us begin with the case $n=1$. Formula (38) implies that the projection in this case is equal to the positive half-current, which can be represented as an integral transform of the total current:

$$
P_{\lambda}^{+}\left(f\left(u_{1}\right)\right)=f_{\lambda}^{+}\left(u_{1}\right)=\oint_{\left|u_{1}\right|>\left|v_{1}\right|} \frac{\mathrm{d} v_{1}}{2 \pi \mathrm{i}} \frac{\theta\left(u_{1}-v_{1}-\lambda\right)}{\theta\left(u_{1}-v_{1}\right) \theta(-\lambda)} f\left(v_{1}\right) .
$$

7 The integral $\oint$ without limits means a formal integral—a continuous extension of the integral over the unit circle. 
The integral kernel of this transform gives the initial condition for the partition function with a factor:

$$
Z^{(1)}\left(u_{1} ; v_{1} ; \lambda\right)=\theta(\hbar) \theta\left(u_{1}-v_{1}\right) \frac{\theta\left(u_{1}-v_{1}-\lambda\right)}{\theta\left(u_{1}-v_{1}\right) \theta(-\lambda)} .
$$

The projections (42) can be calculated by generalizing the method proposed in [1] for the algebra $U_{q}\left(\hat{\mathfrak{s l}}_{2}\right)$. The method uses a recursion over $n$. Let us first present the last total current in (42) as the difference of half-currents:

$$
\begin{gathered}
P_{\lambda-(n-1) \hbar}^{+}\left(f\left(u_{n}\right) \cdots f\left(u_{2}\right) f\left(u_{1}\right)\right)=P_{\lambda-(n-3) \hbar}^{+}\left(f\left(u_{n}\right) \cdots f\left(u_{2}\right)\right) f_{\lambda-(n-1) \hbar}^{+}\left(u_{1}\right) \\
-P_{\lambda-(n-1) \hbar}^{+}\left(f\left(u_{n}\right) \cdots f\left(u_{2}\right) f_{\lambda-(n-1) \hbar}^{-}\left(u_{1}\right)\right) .
\end{gathered}
$$

In the first term we move out the positive half-current from the projection and, therefore, the calculation of this term is reduced to the calculation of $(n-1)$ st projection. In the second term in (44) we move the negative half-current to the left step by step by using the following commutation relation [2]:

$$
f(v) f_{\lambda}^{-}\left(u_{1}\right)=\frac{\theta\left(v-u_{1}-\hbar\right)}{\theta\left(v-u_{1}+\hbar\right)} f_{\lambda+2 \hbar}^{-}\left(u_{1}\right) f(v)+\frac{\theta\left(v-u_{1}+\lambda+\hbar\right)}{\theta\left(v-u_{1}+\hbar\right)} F_{\lambda}(v),
$$

where

$$
F_{\lambda}(v)=\frac{\theta(\hbar)}{\theta(\lambda+\hbar)}\left(f_{\lambda+2 \hbar}^{+}(v) f_{\lambda}^{+}(v)-f_{\lambda+2 \hbar}^{-}(v) f_{\lambda}^{-}(v)\right) .
$$

In each step we obtain an additional term containing $F_{\lambda}(u)$ and in the last step the negative half-current is annihilated by the projection:

$$
P_{\lambda-(n-1) \hbar}^{+}\left(f\left(u_{n}\right) \cdots f\left(u_{2}\right) f_{\lambda-(n-1) \hbar}^{-}\left(u_{1}\right)\right)=\sum_{j=2}^{n} Q_{j}\left(u_{1}\right) X_{j},
$$

where

$$
\begin{aligned}
& Q_{j}(u)=\frac{\theta\left(u_{j}-u+\lambda-(n-2 j+2) \hbar\right)}{\theta\left(u_{j}-u+\hbar\right)} \prod_{k=2}^{j-1} \frac{\theta\left(u_{k}-u-\hbar\right)}{\theta\left(u_{k}-u+\hbar\right)}, \\
& X_{j}=P_{\lambda-(n-1) \hbar}^{+}\left(f\left(u_{n}\right) \cdots f\left(u_{j+1}\right) F_{\lambda-(n-2 j+3) \hbar}\left(u_{j}\right) f\left(u_{j-1}\right) \cdots f\left(u_{2}\right)\right) .
\end{aligned}
$$

Putting $u_{1}=u_{i}$ in (45) we can substitute the negative half-current into the positive one due to the commutation relation for the total currents $f(u)$ and the equality $f(u) f(u)=0$. Moving out the positive half-current to the left one obtains a linear system of equations for $X_{i}, i=2, \ldots, n$ :

$$
P_{\lambda-(n-3) \hbar}^{+}\left(f\left(u_{n}\right) \cdots f\left(u_{2}\right)\right) f_{\lambda-(n-1) \hbar}^{+}\left(u_{i}\right)=\sum_{j=2}^{n} Q_{j}\left(u_{i}\right) X_{j} .
$$

Multiplying each equation (46) by

$$
\frac{\theta\left(u_{i}-u+\lambda\right)}{\theta(\lambda)} \prod_{k=2}^{n} \frac{\theta\left(u_{k}-u_{i}+\hbar\right)}{\theta\left(u_{k}-u+\hbar\right)} \prod_{\substack{k=2 \\ k \neq i}}^{n} \frac{\theta\left(u_{k}-u\right)}{\theta\left(u_{k}-u_{i}\right)},
$$

summing it over $i=2, \ldots, n$ and using the interpolation formula (see appendix A)

$$
Q_{j}(u)=\sum_{i=2}^{n} Q_{j}\left(u_{i}\right) \frac{\theta\left(u_{i}-u+\lambda\right)}{\theta(\lambda)} \prod_{k=2}^{n} \frac{\theta\left(u_{k}-u_{i}+\hbar\right)}{\theta\left(u_{k}-u+\hbar\right)} \prod_{\substack{k=2 \\ k \neq i}}^{n} \frac{\theta\left(u_{k}-u\right)}{\theta\left(u_{k}-u_{i}\right)}
$$


one yields

$$
\begin{aligned}
& P_{\lambda-(n-3) \hbar}^{+}\left(f\left(u_{n}\right) \cdots f\left(u_{2}\right)\right) \sum_{i=2}^{n} \frac{\theta\left(u_{i}-u+\lambda\right)}{\theta(\lambda)} \prod_{k=2}^{n} \frac{\theta\left(u_{k}-u_{i}+\hbar\right)}{\theta\left(u_{k}-u+\hbar\right)} \prod_{\substack{k=2 \\
k \neq i}}^{n} \frac{\theta\left(u_{k}-u\right)}{\theta\left(u_{k}-u_{i}\right)} f_{\lambda-(n-1) \hbar}^{+}\left(u_{i}\right) \\
& =\sum_{j=2}^{n} Q_{j}(u) X_{j} .
\end{aligned}
$$

Comparing (48) with (45) we conclude

$$
\begin{aligned}
& P_{\lambda-(n-1) \hbar}^{+}\left(f\left(u_{n}\right) \cdots f\left(u_{2}\right) f_{\lambda-(n-1) \hbar}^{-}\left(u_{1}\right)\right)=P_{\lambda-(n-3) \hbar}^{+}\left(f\left(u_{n}\right) \cdots f\left(u_{2}\right)\right) \\
& \times \sum_{i=2}^{n} \frac{\theta\left(u_{i}-u_{1}+\lambda\right)}{\theta(\lambda)} \prod_{k=2}^{n} \frac{\theta\left(u_{k}-u_{i}+\hbar\right)}{\theta\left(u_{k}-u_{1}+\hbar\right)} \prod_{\substack{k=2 \\
k \neq i}}^{n} \frac{\theta\left(u_{k}-u_{1}\right)}{\theta\left(u_{k}-u_{i}\right)} f_{\lambda-(n-1) \hbar}^{+}\left(u_{i}\right) .
\end{aligned}
$$

Eventually, returning to formula (44) we derive the following expression for the projection:

$P_{\lambda-(n-1) \hbar}^{+}\left(f\left(u_{n}\right) \cdots f\left(u_{2}\right) f\left(u_{1}\right)\right)=P_{\lambda-(n-3) \hbar}^{+}\left(f\left(u_{n}\right) \cdots f\left(u_{2}\right)\right) f_{\lambda-(n-1) \hbar}^{+}\left(u_{1} ; u_{n}, \ldots, u_{2}\right)$,

where we introduce the linear combination of the currents:

$$
\begin{gathered}
f_{\lambda-(n-2 m+1) \hbar}^{+}\left(u_{m} ; u_{n}, \ldots, u_{m+1}\right)=f_{\lambda-(n-2 m+1) \hbar}^{+}\left(u_{m}\right)-\sum_{i=m+1}^{n} \frac{\theta\left(u_{i}-u_{m}+\lambda+(m-1) \hbar\right)}{\theta(\lambda+(m-1) \hbar)} \\
\times \prod_{k=m+1}^{n} \frac{\theta\left(u_{k}-u_{i}+\hbar\right)}{\theta\left(u_{k}-u_{m}+\hbar\right)} \prod_{\substack{k=m+1 \\
k \neq i}}^{n} \frac{\theta\left(u_{k}-u_{m}\right)}{\theta\left(u_{k}-u_{i}\right)} f_{\lambda-(n-2 m+1) \hbar}^{+}\left(u_{i}\right) .
\end{gathered}
$$

Continuing this calculation by the induction we obtain an expression for the projections in terms of the half-currents (51):

$$
P_{\lambda-(n-1) \hbar}^{+}\left(f\left(u_{n}\right) \cdots f\left(u_{2}\right) f\left(u_{1}\right)\right)=\prod_{n \geqslant m \geqslant 1} f_{\lambda-(n-2 m+1) \hbar}^{+}\left(u_{m} ; u_{n}, \ldots, u_{m+1}\right)
$$

To represent the projections (52) in integral form we first rewrite expression (51) in the form

$$
\begin{aligned}
& f_{\lambda-(n-2 m+1) \hbar}^{+}\left(u_{m} ; u_{n}, \ldots, u_{m+1}\right)=\prod_{k=m+1}^{n} \frac{\theta\left(u_{k}-u_{m}\right) \theta(\hbar)}{\theta\left(u_{k}-u_{m}+\hbar\right)}\left(\prod_{k=m+1}^{n} G_{\hbar}\left(u_{k}-u_{m}\right)\right. \\
& \left.\times f_{\lambda-(n-2 m+1) \hbar}^{+}\left(u_{m}\right)+\sum_{i=m+1}^{n} G_{-\lambda-(m-1) \hbar}\left(u_{m}-u_{i}\right) \prod_{\substack{k=m+1 \\
k \neq i}}^{n} G_{\hbar}\left(u_{k}-u_{i}\right) f_{\lambda-(n-2 m+1) \hbar}^{+}\left(u_{i}\right)\right),
\end{aligned}
$$

where $G_{\lambda}(u-v)=\frac{\theta(u-v+\lambda)}{\theta(u-v) \theta(\lambda)}$. Substituting (37) into (53) and using the addition formula ${ }^{8}$

$$
\sum_{i=1}^{N} \prod_{\substack{j=1 \\ j \neq i}}^{N} G_{\lambda_{j}}\left(u_{j}-u_{i}\right) G_{\lambda_{0}}\left(u_{i}-v\right)=\prod_{i=1}^{N} G_{\lambda_{i}}\left(u_{i}-v\right),
$$

8 This formula can be proved using lemma 2 of appendix A. 
where $\lambda_{0}=\sum_{i=1}^{N} \lambda_{i}$, one can represent the half-currents (51) as an integral transform of the total current:

$$
\begin{aligned}
f_{\lambda-(n-2 m+1) \hbar}^{+}\left(u_{m}\right. & \left.; u_{n}, \ldots, u_{m+1}\right)=\prod_{k=m+1}^{n} \frac{\theta\left(u_{k}-u_{m}\right)}{\theta\left(u_{k}-u_{m}+\hbar\right)} \\
& \times \oint_{\left|u_{i}\right|>|v|} \frac{\mathrm{d} v}{2 \pi \mathrm{i}} \frac{\theta\left(u_{m}-v-\lambda-(m-1) \hbar\right)}{\theta\left(u_{m}-v\right) \theta(-\lambda-(m-1) \hbar)} \prod_{k=m+1}^{n} \frac{\theta\left(u_{k}-v+\hbar\right)}{\theta\left(u_{k}-v\right)} f(v) .
\end{aligned}
$$

Replacing each combination of the half-currents (51) in (52) by their integral form we obtain

$$
\begin{gathered}
P_{\lambda-(n-1) \hbar}^{+}\left(f\left(u_{n}\right) \cdots f\left(u_{2}\right) f\left(u_{1}\right)\right)=\prod_{n \geqslant k>m \geqslant 1} \frac{\theta\left(u_{k}-u_{m}\right)}{\theta\left(u_{k}-u_{m}+\hbar\right)} \oint_{\left|u_{i}\right|>\left|v_{j}\right|} \frac{\mathrm{d} v_{n} \cdots \mathrm{d} v_{1}}{(2 \pi \mathrm{i})^{n}} \\
\times \prod_{n \geqslant k>m \geqslant 1} \frac{\theta\left(u_{k}-v_{m}+\hbar\right)}{\theta\left(u_{k}-v_{m}\right)} \prod_{m=1}^{n} \frac{\theta\left(u_{m}-v_{m}-\lambda-(m-1) \hbar\right)}{\theta\left(u_{m}-v_{m}\right) \theta(-\lambda-(m-1) \hbar)} f\left(v_{n}\right) \cdots f\left(v_{1}\right) .
\end{gathered}
$$

Formulae (52) and (56) yield expressions for the universal elliptic weight functions in terms of the current generators of the algebra $\mathcal{A}$.

\subsection{Universal weight function and the SOS model partition function}

To extract the integral kernel from expression (56) and derive a formula for the partition function we use the Hopf pairing (35). Let us calculate the following expression generalizing (43):

$$
\begin{gathered}
Z^{(n)}\left(u_{n}, \ldots, u_{1} ; v_{n}, \ldots, v_{1} ; \lambda\right)=\prod_{i, j=1}^{n} \theta\left(u_{i}-v_{j}\right) \prod_{n \geqslant k>m \geqslant 1} \frac{\theta\left(u_{k}-u_{m}+\hbar\right) \theta\left(v_{k}-v_{m}-\hbar\right)}{\theta\left(u_{k}-u_{m}\right) \theta\left(v_{k}-v_{m}\right)} \\
\times(\hbar \theta(\hbar))^{n}\left\langle P_{\lambda-(n-1) \hbar}^{+}\left(f\left(u_{n}\right) \cdots f\left(u_{1}\right)\right), e\left(v_{n}\right) \cdots e\left(v_{1}\right)\right\rangle .
\end{gathered}
$$

Using the expression for the projection of the product of the total currents (56) and formula (36) we obtain

$$
\begin{aligned}
& Z^{(n)}\left(u_{n}, \ldots, u_{1} ; v_{n}, \ldots, v_{1} ; \lambda\right)=\theta(\hbar)^{n} \prod_{i, j=1}^{n} \theta\left(u_{i}-v_{j}\right) \prod_{k>m} \frac{\theta\left(v_{k}-v_{m}-\hbar\right)}{\theta\left(v_{k}-v_{m}\right)} \\
& \times \sum_{\sigma \in S_{n}} \prod_{\substack{l<l^{\prime} \\
\sigma(l)>\sigma\left(l^{\prime}\right)}} \frac{\theta\left(v_{\sigma(l)}-v_{\sigma\left(l^{\prime}\right)}+\hbar\right)}{\theta\left(v_{\sigma(l)}-v_{\sigma\left(l^{\prime}\right)}-\hbar\right)} \\
& \times \prod_{k>m} \frac{\theta\left(u_{k}-v_{\sigma(m)}+\hbar\right)}{\theta\left(u_{k}-v_{\sigma(m)}\right)} \prod_{m=1}^{n} \frac{\theta\left(u_{m}-v_{\sigma(m)}-\lambda-(m-1) \hbar\right)}{\theta\left(u_{m}-v_{\sigma(m)}\right) \theta(-\lambda-(m-1) \hbar)} \\
& =\prod_{k>m} \frac{\theta\left(v_{k}-v_{m}-\hbar\right)}{\theta\left(v_{k}-v_{m}\right)} \sum_{\sigma \in S_{n}} \prod_{\substack{l<l^{\prime} \\
\sigma(l)>\sigma\left(l^{\prime}\right)}} \frac{\theta\left(v_{\sigma(l)}-v_{\sigma\left(l^{\prime}\right)}+\hbar\right)}{\theta\left(v_{\sigma(l)}-v_{\sigma\left(l^{\prime}\right)}-\hbar\right)} \\
& \times \prod_{k>m} \theta\left(u_{k}-v_{\sigma(m)}+\hbar\right) \prod_{k<m} \theta\left(u_{k}-v_{\sigma(m)}\right) \\
& \times \prod_{m=1}^{n} \frac{\theta\left(u_{m}-v_{\sigma(m)}-\lambda-(m-1) \hbar\right) \theta(\hbar)}{\theta(-\lambda-(m-1) \hbar)} .
\end{aligned}
$$

We see from this formula that expression (58) defines a holomorphic function of the variables $u_{i}$. 
Theorem 1. The set of functions $\left\{Z^{(n)}\left(u_{n}, \ldots, u_{1} ; v_{n}, \ldots, v_{1} ; \lambda\right)\right\}_{n \geqslant 1}$ defined by formula (57) satisfies the conditions of the propositions 1, 2, 3 and the initial condition (22). Therefore, by virtue of theorem 1 they coincide with the partition functions of the SOS model with DWBC:

$$
\begin{aligned}
& Z_{-+}^{+-}\left(u_{n}, \ldots, u_{1} ; v_{n}, \ldots, v_{1} ; \lambda\right)=\prod_{n \geqslant k>m \geqslant 1} \frac{\theta\left(v_{k}-v_{m}-\hbar\right)}{\theta\left(v_{k}-v_{m}\right)} \\
& \quad \times \sum_{\sigma \in S_{n}} \prod_{\substack{l<l^{\prime} \\
\sigma(l)>\sigma\left(l^{\prime}\right)}} \frac{\theta\left(v_{\sigma(l)}-v_{\sigma\left(l^{\prime}\right)}+\hbar\right)}{\theta\left(v_{\sigma(l)}-v_{\sigma\left(l^{\prime}\right)}-\hbar\right)} \prod_{1 \leqslant k<m \leqslant n} \theta\left(u_{k}-v_{\sigma(m)}\right) \\
& \times \prod_{n \geqslant k>m \geqslant 1} \theta\left(u_{k}-v_{\sigma(m)}+\hbar\right) \prod_{m=1}^{n} \frac{\theta\left(u_{m}-v_{\sigma(m)}-\lambda-(m-1) \hbar\right) \theta(\hbar)}{\theta(-\lambda-(m-1) \hbar)} .
\end{aligned}
$$

The initial condition (22) is verified by checking formula (43). The first factor on the right-hand side of (57) is symmetric with respect to both sets of variables. Then the symmetry with respect to the variables $\{u\}$ and the variables $\{v\}$ follows from the commutation relations (29) and (28) respectively. Formula (58) implies that (57) are elliptic polynomials of degree $n$ with character (18) in variables $u_{i}$, particularly in $u_{n}$. Now let us substitute $u_{n}=v_{n}-\hbar$ into (58). The non-vanishing terms on the right-hand side correspond to the permutations $\sigma \in S_{n}$ satisfying $\sigma(n)=n$. Substituting $u_{n}=v_{n}-\hbar$ into these terms one obtains the recurrent relation (19).

Remark 5. We do not want to recall here a definition of elliptic weight function from [11]. We observe only that our formula (57) is a specification of their definition (formula (2.30) and lemma 2.31, p 21 of [11]) for special values of parameters. We should also stress that we use here additive notations for theta-functions when the definition in [11] works with the multiplicative ones. The same description is used in [10] where the identification is also achieved. We hope that the advantage of the projection method we have used here is its universality.

\section{Trigonometric limit}

In this section, we investigate the trigonometric degenerations of the formulae obtained in the elliptic case. In particular, taking the corresponding trigonometric limit in the expression for the SOS model partition function (59) we reproduce the expression for the six-vertex partition function (5).

First we consider the degeneration of $R$-matrix - the matrix of the Boltzmann weights, which defines the model. To do it we need the formula of the trigonometric degeneration $(\tau \rightarrow \mathrm{i} \infty$ ) of the odd theta function defined by the conditions theta:

$$
\lim _{\tau \rightarrow \mathrm{i} \infty} \theta(u)=\frac{\sin \pi u}{\pi} .
$$

In terms of the multiplicative variables $z=\mathrm{e}^{2 \pi \mathrm{i} u}, w=\mathrm{e}^{2 \pi \mathrm{i} v}$ this formula can be rewritten as follows:

$$
2 \pi \mathrm{i} \mathrm{e}^{\pi \mathrm{i}(u+v)} \lim _{\tau \rightarrow \mathrm{i} \infty} \theta(u-v)=z-w .
$$

Multiplying the $R$-matrix (10) by $2 \pi \mathrm{i} \mathrm{e}^{\pi \mathrm{i}(u+v)}$ and taking the limit we obtain the following matrix depending rationally on the multiplicative variables $z, w$ and multiplicative parameters $q=\mathrm{e}^{\pi \mathrm{i} \hbar}, \mu=\mathrm{e}^{2 \pi \mathrm{i} \lambda}$ : 


$$
\begin{aligned}
R(z, w ; \mu)= & 2 \pi \mathrm{i}^{\pi \mathrm{i}(u+v)} \lim _{\tau \rightarrow \mathrm{i} \infty} R(u-v ; \lambda) \\
= & \left(\begin{array}{cccc}
z q-w q^{-1} & 0 & 0 & 0 \\
0 & \frac{(z-w)\left(\mu q-q^{-1}\right)}{(\mu-1)} & \frac{(z-w \mu)\left(q-q^{-1}\right)}{(1-\mu)} & 0 \\
0 & \frac{(z \mu-w)\left(q-q^{-1}\right)}{(\mu-1)} & \frac{(z-w)\left(\mu q^{-1}-q\right)}{(\mu-1)} & 0 \\
0 & 0 & 0 & \left(z q-w q^{-1}\right)
\end{array}\right) .
\end{aligned}
$$

The matrix (60) inherits the property to satisfy the dynamical Yang-Baxter equation and it defines the statistical model which is called the trigonometric SOS model.

To obtain the non-dynamical trigonometric case we need to implement the additional limit $\lambda \rightarrow-\mathrm{i} \infty$ implying $\mu \rightarrow \infty$ (or $\lambda \rightarrow \mathrm{i} \infty$ implying $\mu \rightarrow 0$ ):

$$
\tilde{R}(z, w)=\lim _{\mu \rightarrow \infty} R(z, w ; \mu)=\left(\begin{array}{cccc}
z q-w q^{-1} & 0 & 0 & 0 \\
0 & q(z-w) & \left(q-q^{-1}\right) w & 0 \\
0 & \left(q-q^{-1}\right) z & q^{-1}(z-w) & 0 \\
0 & 0 & 0 & z q-w q^{-1}
\end{array}\right) .
$$

The matrix (61) differs from the matrix of six-vertex Boltzmann weights (2) by the transformation (21). Taking into account the remark 4 (from section 3.2) we conclude that both matrices (2) and (61) define the same partition function $Z(\{z\},\{w\})$ with $\mathrm{DWBC}^{9}$.

To obtain the partition function with DWBC for the trigonometric SOS model one should multiply the partition function with DWBC for the elliptic SOS model by a certain factor and take the trigonometric limit:

$$
\begin{aligned}
Z_{-+}^{+-}(\{z\},\{w\} ; \mu)= & \prod_{k, j=1}^{n}\left(2 \pi \mathrm{i}^{\pi \mathrm{i}\left(u_{k}+v_{j}\right)}\right) \lim _{\tau \rightarrow \mathrm{i} \infty} Z_{-+}^{+-}(\{u\},\{v\} ; \lambda) \\
= & \prod_{n \geqslant k>m \geqslant 1} \frac{w_{k} q^{-1}-w_{m} q}{w_{k}-w_{m}} \sum_{\sigma \in S_{n}} \prod_{\substack{l<l^{\prime} \\
\sigma(l)>\sigma\left(l^{\prime}\right)}} \frac{w_{\sigma(l)} q-w_{\sigma\left(l^{\prime}\right)} q^{-1}}{w_{\sigma(l)} q^{-1}-w_{\sigma\left(l^{\prime}\right)} q} \\
& \times \prod_{n \geqslant k>m \geqslant 1}\left(z_{k} q-w_{\sigma(m)} q^{-1}\right) \prod_{1 \leqslant k<m \leqslant n}\left(z_{k}-w_{\sigma(m)}\right) \\
& \times \prod_{m=1}^{n} \frac{\left(z_{m}-w_{\sigma(m)} \mu q^{2(m-1)}\right)\left(q-q^{-1}\right)}{\left(1-\mu q^{2(m-1)}\right)}
\end{aligned}
$$

It is easy to check that formula (5) is obtained from formula (62) by taking the limit: $Z(\{z\},\{w\})=\lim _{\mu \rightarrow \infty} Z_{-+}^{+-}(\{z\},\{w\} ; \mu)$.

\section{Acknowledgements}

This paper is a part of a PhD thesis of AS which he is preparing in co-direction of SP and $\mathrm{V} \mathrm{R}$ in the Bogoliubov Laboratory of Theoretical Physics, JINR, Dubna and in LAREMA, Département de Mathématics, Université d'Angers. He is grateful to the CNRS-Russia exchange program on mathematical physics and personally to J-M Maillet for financial and general support of this thesis project. V R is thankful to Ph Di Franchesco and T Miwa for their stimulating lectures and their interest during ENIGMA School 'Quantum Integrability' held in Lalonde les Maures on 14-19 October, 2007. He thanks O Babelon and M Talon for

\footnotetext{
9 The matrix (61) is a limit of a matrix which differs from (10) by the transformation (21) with $\rho=q$.
} 
the invitation to this school. V R had used during the project a partial financial support by ANR GIMP and a support of INFN-RFBR ‘Einstein' grant (Italy-Russia). SP was supported in part by RFBR grant 06-02-17383. Both V R and S P were supported in part by the grant for support of scientific schools NSh-8065.2006.2.

\section{Appendix A. Interpolation formula for the elliptic polynomials}

We will call by a character a group homomorphism $\chi: \Gamma \rightarrow \mathbb{C}^{\times}$, where $\Gamma=\mathbb{Z}+\tau \mathbb{Z}$ and $\mathbb{C}^{\times}$is a multiplicative group of nonzero complex numbers. Each character $\chi$ and an integer number $n$ define a space $\Theta_{n}(\chi)$ consisting of the holomorphic functions on $\mathbb{C}$ with the translation properties

$$
\phi(u+1)=\chi(1) \phi(u), \quad \phi(u+\tau)=\chi(\tau) \mathrm{e}^{-2 \pi \mathrm{i} n u-\pi \mathrm{i} n \tau} \phi(u) .
$$

If $n>0$ then $\operatorname{dim} \Theta_{n}(\chi)=n$ (and $\operatorname{dim} \Theta_{n}(\chi)=0$ if $n<0$ ). The elements of the space $\Theta_{n}(\chi)$ are called elliptic polynomials (or theta-functions) of degree $n$ with the character $\chi$ (see [13]).

Proposition 4. Let $\left\{\phi_{j}\right\}_{j=1}^{n}$ be a basis of $\Theta_{n}(\chi)$, with the character $\chi(1)=(-1)^{n}, \chi(\tau)=$ $(-1)^{n} \mathrm{e}^{2 \pi \mathrm{i} \alpha}$, then the determinant of the matrix $\left\|\phi_{j}\left(u_{i}\right)\right\|_{1 \leqslant i, j \leqslant n}$ is equal to

$$
\operatorname{det}\left\|\phi_{j}\left(u_{i}\right)\right\|=C \cdot \theta\left(\sum_{k=1}^{n} u_{k}-\alpha\right) \prod_{i<j} \theta\left(u_{i}-u_{j}\right),
$$

where $C$ is a nonzero constant.

Consider the ratio

$$
\frac{\operatorname{det}\left\|\phi_{j}\left(u_{i}\right)\right\|}{\theta\left(\sum_{k=1}^{n} u_{k}-\alpha\right) \prod_{i<j} \theta\left(u_{i}-u_{j}\right)} .
$$

This is an elliptic function of each $u_{i}$ with only simple pole in any fundamental domain (the points $u_{i}$ satisfying $\sum_{k=1}^{n} u_{k}-\alpha \in \Gamma$ ). Therefore, it is a constant function of each $u_{i}$. Thus this ratio does not depend on each $u_{i}$ and we have to prove that it does not vanish, that is to prove that the determinant $\operatorname{det}\left\|\phi_{j}\left(u_{i}\right)\right\|$ is not identically zero. Let us denote by $\Delta_{j_{1}, \ldots, j_{k}}^{i_{1}, \ldots, i_{k}}$ the minor of this determinant corresponding to the $i_{1}$ th $, \ldots, i_{k}$ th rows and the $j_{1}$ th, $\ldots, j_{k}$ th columns. Suppose that this determinant is identically zero and consider the following decomposition:

$$
\operatorname{det}\left\|\phi_{j}\left(u_{i}\right)\right\|=\sum_{k=1}^{n}(-1)^{k+1} \phi_{k}\left(y_{1}\right) \Delta_{1, \ldots, k-1, k+1, \ldots, n}^{2, \ldots, n} .
$$

Since the functions $\phi_{k}\left(y_{1}\right)$ are linearly independent the minors $\Delta_{1, \ldots, k-1, k+1, \ldots, n}^{2, \ldots, n}$ are identically zero. Decomposing the minor $\Delta_{2, \ldots, n}^{2, \ldots, n}$ we conclude that the minors $\Delta_{2, \ldots, k-1, k+1, \ldots, n}^{3, \ldots, n}$ are identically zero, and so on. Finally, we obtain that $\Delta_{n}^{n}=\phi_{n}\left(y_{n}\right)$ is identically zero which cannot be true.

Lemma 2. Let us consider two elliptic polynomials $P_{1}, P_{2} \in \Theta_{n}(\chi)$, where $\chi(1)=$ $(-1)^{n}, \chi(\tau)=(-1)^{n} \mathrm{e}^{\alpha}$, and $n$ points $u_{i}, i=1, \ldots, n$, such that $u_{i}-u_{j} \notin \Gamma, i \neq j$, and $\sum_{k=1}^{n} u_{k}-\alpha \notin \Gamma$. If the values of these polynomials coincide at these points, $P_{1}\left(u_{i}\right)=P_{2}\left(u_{i}\right)$, then these polynomials coincide identically: $P_{1}(u)=P_{2}(u)$.

Decomposing the considering polynomials as $P_{a}(u)=\sum_{i=1}^{n} p_{a}^{i} \phi_{i}(u), a=1,2$, we have the system of equations

$$
\sum_{i=1}^{n} p_{12}^{i} \phi_{i}(u)=0
$$


with respect to the variables $p_{12}^{i}=p_{1}^{i}-p_{2}^{i}$. As we have proved, the determinant of this system is equal to (A.1) and therefore is not zero. Hence, this system has only a trivial solution $p_{12}^{i}=0$, but this implies $P_{1}(u)=P_{2}(u)$.

Let $P \in \Theta_{n}(\chi)$ be an elliptic polynomial, where $\chi(1)=(-1)^{n}, \chi(\tau)=(-1)^{n} \mathrm{e}^{2 \pi \mathrm{i} \alpha}$, and $u_{i}, i=1, \ldots, n$, be $n$ points such that $u_{i}-u_{j} \notin \Gamma, i \neq j$, and $\sum_{k=1}^{n} u_{k}-\alpha \notin \Gamma$. This polynomial can be restored by the values at these points:

$P(u)=\sum_{i=1}^{n} P\left(u_{i}\right) \frac{\theta\left(u_{i}-u+\alpha-\sum_{m=1}^{n} u_{m}\right)}{\theta\left(\alpha-\sum_{m=1}^{n} u_{m}\right)} \prod_{\substack{k=1 \\ k \neq i}}^{n} \frac{\theta\left(u_{k}-u\right)}{\theta\left(u_{k}-u_{i}\right)}$.

Indeed, the right-hand side belongs to $\Theta_{n}(\chi)$, this equality holds at points $u=u_{i}$. Using lemma 2 we conclude that (A.4) holds at all $u \in \mathbb{C}$.

Consider the meromorphic functions

$$
Q_{j}(u)=\frac{\theta\left(u_{j}-u+\lambda-(n-2 j+2) \hbar\right)}{\theta\left(u_{j}-u+\hbar\right)} \prod_{k=2}^{j-1} \frac{\theta\left(u_{k}-u-\hbar\right)}{\theta\left(u_{k}-u+\hbar\right)} .
$$

It is easy to check that the functions

$$
\begin{aligned}
P_{j}(u) & =\prod_{k=2}^{n} \theta\left(u_{k}-u+\hbar\right) Q_{j}(u) \\
& =\theta\left(u_{j}-u+\lambda-(n-2 j+2) \hbar\right) \prod_{k=2}^{j-1} \theta\left(u_{k}-u-\hbar\right) \prod_{k=j+1}^{n} \theta\left(u_{k}-u+\hbar\right)
\end{aligned}
$$

belong to $\Theta_{n-1}(\chi)$, where $\chi(1)=(-1)^{n-1}, \chi(\tau)=(-1)^{n-1} \mathrm{e}^{2 \pi \mathrm{i} \alpha}, \alpha=\lambda+\sum_{k=2}^{n} u_{k}$. Since $\lambda \notin \Gamma$, the polynomials $P_{j}(u)$ can be restored by their values $P_{j}\left(u_{i}\right)$ via the interpolation formula (A.4). Taking into account the relation between $Q_{j}(u)$ and $P_{j}(u)$ we obtain formula (47). ${ }^{10}$

\section{Appendix B. Proof of formula (36)}

Let $\mathcal{A}_{f}^{n}$ be a subspace spanned by $\hat{f}\left[s_{n}\right] \cdots \hat{f}\left[s_{1}\right]$ (that is by (40), where $s_{n}, \ldots, s_{1} \in \mathcal{K}_{0}$. Note first that due to the Hopf properties of the pairing (35) the current $e(u)$ annihilates the spaces $\mathcal{A}_{f}^{k} H^{+}$for $k \geqslant 2$. Indeed, using the formulae $\langle x y, z\rangle=\langle x \otimes y, \Delta(z)\rangle,\langle x, 1\rangle=\varepsilon(x)$ and (32) we obtain $\left\langle\mathcal{A}_{f}^{k} H^{+}, e(u)\right\rangle \subset\left\langle\mathcal{A}_{f}^{1} \otimes \mathcal{A}_{f}^{k-1} H^{+}, e(u) \otimes 1+K^{-}(u) \otimes e(u)\right\rangle=\{0\}$. It follows from formula (33) that the opposite coproduct on $\mathcal{A}_{f}^{n}$ has the form

$$
\begin{aligned}
\Delta^{o p}\left(f\left(t_{n}\right) \cdots\right. & \left.f\left(t_{1}\right)\right) \in H^{+} \otimes \mathcal{A}_{f}^{n}+\sum_{k=2}^{n} \mathcal{A}_{f}^{k} H^{+} \otimes \mathcal{A}_{f}^{n-k}+ \\
& +\sum_{j=1}^{n} \prod_{i=j+1}^{n} K^{+}\left(t_{i}\right) f\left(t_{j}\right) \prod_{i=1}^{j-1} K^{+}\left(t_{i}\right) \otimes f\left(t_{n}\right) \cdots f\left(t_{j+1}\right) f\left(t_{j-1}\right) \cdots f\left(t_{1}\right) .
\end{aligned}
$$

Now let us prove formula (36) by induction. For $n=1$ it coincides with the definition of the pairing. Suppose that it holds for $n-1$. Then using the formulae $\langle x, y z\rangle=$ $\left\langle\Delta^{o p}(x), y \otimes z\right\rangle,\langle 1, x\rangle=\varepsilon(x)$ and the commutation relation (27) we obtain

\footnotetext{
${ }^{10}$ We can suppose the condition $u_{i}-u_{j} \notin \Gamma$, because $u_{i}$ in formula (47) are formal variables.
} 


$$
\begin{aligned}
\left\langle f\left(t_{n}\right) \cdots f\left(t_{1}\right), e\left(v_{n}\right) \cdots e\left(v_{1}\right)\right\rangle=\left\langle\Delta^{o p}\left(f\left(t_{n}\right) \cdots f\left(t_{1}\right)\right), e\left(v_{n}\right) \otimes e\left(v_{n-1}\right) \cdots e\left(v_{1}\right)\right\rangle \\
=\hbar^{-n} \sum_{j=1}^{n} \prod_{l^{\prime}=j+1}^{n} \frac{\theta\left(v_{n}-v_{\sigma\left(l^{\prime}-1\right)}+\hbar\right)}{\theta\left(v_{n}-v_{\sigma\left(l^{\prime}-1\right)}-\hbar\right)} \delta\left(t_{j}, v_{n}\right) \\
\quad \times \sum_{\sigma \in S_{n-1}} \prod_{\substack{1 \leqslant l<l^{\prime} \leqslant n-1 \\
\sigma(l)>\sigma\left(l^{\prime}\right)}} \frac{\theta\left(v_{\sigma(l)}-v_{\sigma\left(l^{\prime}\right)}+\hbar\right)}{\theta\left(v_{\sigma(l)}-v_{\sigma\left(l^{\prime}\right)}-\hbar\right)} \prod_{m=1}^{j-1} \delta\left(t_{m}, v_{\sigma(m)}\right) \prod_{m=j+1}^{n} \delta\left(t_{m}, v_{\sigma(m-1)}\right) .
\end{aligned}
$$

Taking into account the identity

$$
\sum_{\sigma \in S_{n}} X(\sigma)=\sum_{j=1}^{n} \sum_{\sigma \in S_{n-1}} X\left(\begin{array}{ccccccc}
1 & \ldots & j-1 & j & j+1 & \ldots & n \\
\sigma(1) & \ldots & \sigma(j-1) & n & \sigma(j) & \ldots & \sigma(n-1)
\end{array}\right)
$$

one yields formula (36).

\section{References}

[1] Khoroshkin S and Pakuliak S 2005 Theor. Math. Phys. $1451373-99$

[2] Enriquez B and Felder G 1998 Commun. Math. Phys. 195 651-89

[3] Enriquez B and Rubtsov R 1997 Ann. Sci. Ecole Norm. Sup. 30 821-46

[4] Enriquez B and Rubtsov V 1999 Isr. J. Math. 112 61-108

[5] Enriquez B and Rubtsov V 1999 Am. Math. Soc. Transl. Ser. 191 33-65

[6] Drinfeld V 1988 Sov. Math. Doc. 36 212-16

[7] Ding J, Khroshkin S and Pakuliak S 2000 Theor. Math. Phys. 124 1007-37

[8] Enriquez B, Khoroshkin S and Pakuliak S 2007 Commun. Math. Phys. 276 691-725

[9] Igerzin A 1987 Sov. Phys.-Dokl. 32 878-79

[10] Rosengren H 2008 An Izergin-Korepin-type identity for the 8VSOS model, with applications to alternating sign matrices Preprint arXiv:0801.1229

[11] Tarasov V and Varchenko A 1997 Astérisque 246135 (ed SMF)

[12] Khoroshkin S, Pakuliak S and Tarasov V 2007 J. Geom. Phys. 57 1713-32

[13] Felder G and Schorr A 1999 J. Phys. A: Math. Gen. 32 8001-22

[14] Date E, Jimbo M, Kuniba A, Miwa T and Okado M 1987 Nucl. Phys. B 290 231-73

[15] Pakuliak S, Rubtsov V and Silantyev A 2008 Classical elliptic current algebras I,II J. Generalized Lie Theor. App. 2 65-78 and 79-93

[16] Kuperberg G 1996 Int. Math. Res. Not. 10 139-50

[17] Odesskii A and Feigin B 1997 Int. Math. Res. Not. 11 531-39

[18] Felder G, Varchenko A and Tarasov V 1997 Topics in Singularity Theory (Am. Math. Soc. Transl. Ser. vol 180) (Providence, RI: American Mathematical Society) pp 45-75

[19] Enriquez B, Pakuliak S and Rubtsov V Contemp. Math. 433 177-90

[20] Korepin V 1982 Commun. Math. Phys. 86 391-418 